\title{
MODELING OF ELECTRON CYCLOTRON RESONANCE ION SOURCE PLASMAS *
}

\author{
D. H. Edgell ${ }^{\dagger}$, J. S. Kim and I. N. Bogatu, FARTECH, Inc., San Diego, CA 92121, USA \\ R. C. Pardo and R. C. Vondrasek, ANL, Argonne, IL 604390, USA
}

\section{Abstract}

A computer model for both electrons and multiple ion species in an Electron Cyclotron Resonance Ion Source (ECRIS) plasma that is 1D spatially has been developed. The non-Maxwellian anisotropic electron-distributionfunction (EDF) is modeled using a 1D axially bounceaveraged Fokker-Planck code that is $2 \mathrm{D}$ in velocity space (velocity and pitch angle). ECR heating is treated using a quasi-linear rf-diffusion term including relativistic detuning and $\mathrm{rf}$ pitch-angle scattering. Under typical ECRIS conditions, the electrons are very non-collisional and magnetically confined. Thus, the ions must follow the electron confinement via the electrostatic potential. The ion species are assumed to be highly collisionally coupled and are treated using a 1D fluid model characterized by a single fluid velocity. Ion charge-state-distributions (CSD) flowing into the extraction aperture can be calculated and used as input to an ion beam extraction code. The modeling reproduces several experimentally observed characteristics of ECRIS plasmas.

\section{INTRODUCTION}

Optimization of an Electron Cyclotron Resonance Ion Source (ECRIS) to produce high currents of high charge state ions is typically a trial and error process. A better understanding of the physics in the core plasma and the ions flowing into the extraction region would aid in ECRIS design and optimization. This complex task requires consideration of magnetic mirror and multipole confinement; rf heating and a resultant non-Maxwellian and anisotropic electron-distribution-function (EDF); multiple ion atomic species each with its own chargestate-distribution (CSD); an axially varying electrostatic plasma potential, etc.

Simple zero dimensional (0D) models ignoring some of the above can be successful at matching experiment CSDs $[1,2]$, but require trial and error guesses at parameters such as the electron density, $n_{e}$, assumed a Maxwellian EDF with an electron temperature, $T_{e}$, etc. A predictive model should rely on experimental "knobs" such as magnetic field and rf power to calculate the plasma parameters and CSD.

A predictive one-dimensional (1D) "General ECRIS Model" (GEM) [3] is under development by FARTECH, Inc. to model an ECRIS plasma. GEM is successful in predicting several features typical of an ECRIS.

\footnotetext{
* Work at FARTECH supported by the U.S. DOE-SBIR Grant Number DE-FG03-97ER82381. Work at ANL supported by the U.S. DOE, Nuclear Physics Division, under contract W-31-109-ENG-38.
}

†edgell@far-tech.com

\section{PLASMA MODELING}

GEM is a multi-species 1D code for the modeling of an ECRIS. It takes into account both ionization, including the excitation-autoionization process in the collision cross-sections [4], and charge-exchange. GEM consists of two major parts discussed separately below: the 1D bounce-averaged Fokker-Planck (FP) modeling of the electrons and the 1D fluid modeling of the ions.

\subsection{Electron Fokker-Planck Modeling}

Rather than assuming arbitrary Maxwellian temperatures as common in many 0D models, GEM calculates the EDF by solving the Fokker-Planck equation using the nonlinear multi-species code FPPAC94 [5]. As the electrons are quite collisionless, it is appropriate to extend the calculations to include 1D effects by bounceaveraging the Fokker-Planck Coefficients. Bounceaveraging includes the effects of axial variations in plasma parameter profiles by averaging these effects over the entire path of an electron "bouncing" back and forth inside a magnetic well. GEM uses the bounce-averaging routines of BACON11 [6] modified to accept collisions with multiple ion species and asymmetric magnetic wells such as common in an ECRIS.

Electron Cyclotron Resonance Heating is treated using a quasi-linear rf diffusion coefficient. This coefficient includes the effects of relativistic detuning of the resonance and rf pitch angle scattering which limits confinement by scattering very fast electrons. Bounceaveraging of the rf coefficients allows the model to take into account the axial location and width of the rf resonance. The absolute magnitude of the coefficient is adjusted to match the total deposited power from the model with the experimentally launched power.

A typical EDF calculated by GEM is shown in Figures 1 and 2. The ECRIS geometry for this run is based on ECR-II [7] at Argonne National Laboratory (ANL) where for simplicity a symmetric magnetic field of mirror ratio was used. Based on ECR-II runs, a deposited rf power of about $540 \mathrm{~W}$ and a neutral oxygen gas pressure of $7 \cdot 10^{-7}$ torr were chosen. The EDF is highly non-Maxwellian as expected in an ECRIS. It can be seen that the EDF peaks with pitch angle at about $65^{\circ}$. Electrons with higher pitch angles cannot reach the $\mathrm{rf}$ resonance due to their strong magnetic trapping.

Electron density and energy axial profiles can be determined by mapping the bounce-averaged EDF to each axial position, taking into account the magnetic and potential profiles. Figures 3 and 4 show the density and 
average electron energy profiles, respectively, that correspond to the EDF given in Figures 1 and 2. One can see that GEM predicts a hot dense plasma core typical of an ECRIS. The electron energy peaks just outside the rf resonance, similar perhaps to the hot electron ring sometimes seen outside the core of an ECRIS.

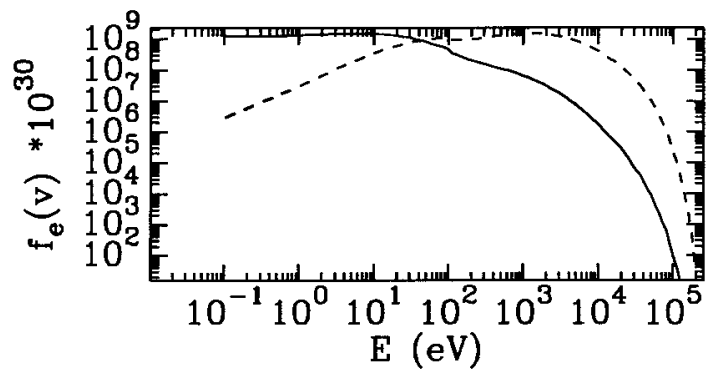

Figure 1: Typical EDF vs. electron energy. The solid line is the $f_{e}(\mathrm{v})$. The dotted line is $f_{e}(\mathrm{v}) d \mathrm{v}$, which is an indication of the total number of electrons at $\mathrm{v}$.

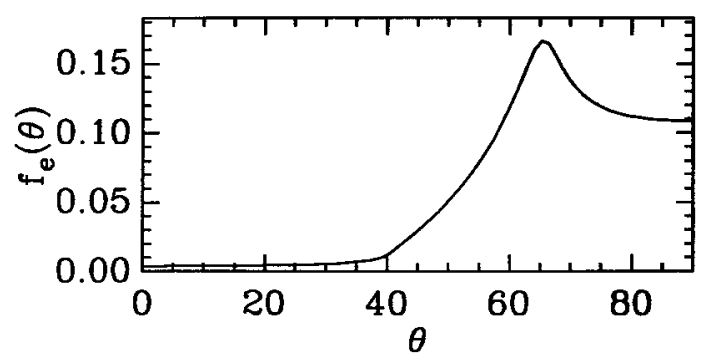

Figure 2: Typical EDF vs. pitch angle. The peak is located at the pitch angle required to just reach the $\mathrm{rf}$ resonance.

\subsection{Ion Fluid Modeling}

Unlike the electrons, the ions in an ECRIS are highly collisional and a fluid model is appropriate for treating them. All ions are assumed to flow at the same ion fluid velocity, $u_{i}$, due to frictional drag from their high collisionallity. Each ion of atomic species $j$ and charge $q$ is then governed by an ion continuity equation

$$
\frac{\partial n_{j, q}(z, t)}{\partial t}=S_{j, q}(z, t)-\frac{1}{A(z)} \frac{\partial}{\partial z}\left(A(z) n_{j, q}(z, t) u_{i}(z, t)\right)
$$

where $n_{j, q}$ is the ion density and $S_{j, q}$ is the ion source/sink term due to ionization and charge-exchange events. $\mathrm{A}(\mathrm{z})$ is the plasma cross-sectional area of the ion flux tube which is inversely proportional to the magnetic field. When the ion continuity equations are summed for all ions one gets

$$
\begin{aligned}
\frac{\partial n_{e}(z, t)}{\partial t}=S_{e}(z, t)-n_{e}(z, t) & \frac{\partial u_{i}(z, t)}{\partial z}-u_{i}(z, t) \frac{\partial n_{e}(z, t)}{\partial z} \\
& -\frac{n_{e}(z, t) u_{i}(z, t)}{A(z)} \frac{\partial}{\partial z}[A(z)]
\end{aligned}
$$

where $S_{e}$ is the total electron source and quasineutrality

$$
n_{e}(z, t)=\sum_{j, q} q n_{j, q}(z, t)
$$

is assumed. This almost appears to be an electron continuity equation, however, $u_{i}$, should not be confused with an electron fluid velocity as the electrons are definitely too collisionless to be fluid-like. Boundary conditions, either free-flow or flow at the sound speed (the Bohm criterion) can be enforced at the device edges.

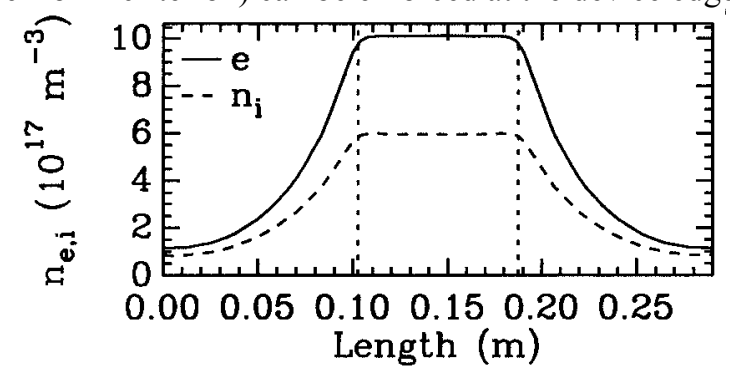

Figure 3: Typical electron density profile (solid line) and total ion density profile (dashed line). Dotted lines show location of $\mathrm{rf}$ resonance.

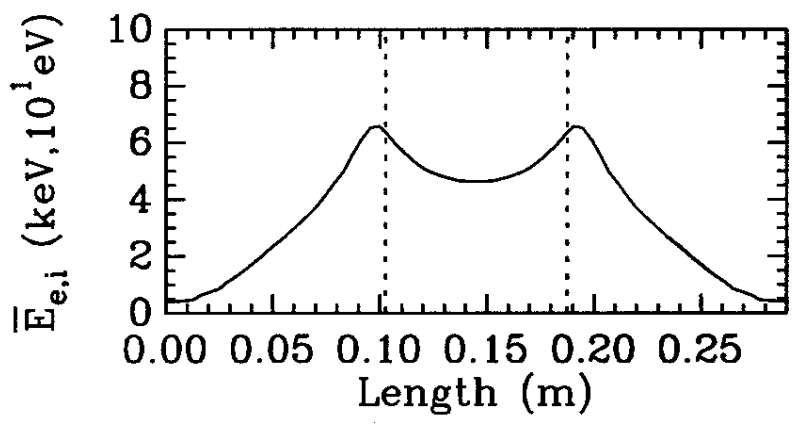

Figure 4: Typical average electron energy profile. Dotted lines show location of $r f$ resonance.

GEM solves the above total ion continuity equation for the $u_{i}$ that is required to match the Fokker-Planck modeled electron density and source terms. This fluid velocity is then used in each individual ion continuity equation and the ion CSD at each axial location is adjusted accordingly.

Now the sum of the ion momentum fluid equations

$$
\begin{array}{r}
\left(\sum_{j, q} m_{j} n_{j, q}(z, t)\right) \frac{\partial u_{i}(z)}{\partial t}=-\left(\sum_{j, q} q n_{j, q}(z, t)\right) e \nabla \varphi(z, t) \\
-\left(\sum_{j, q} m_{j} n_{j, q}(z, t)\right) u_{i}(z, t) \frac{\partial u_{i}(z, t)}{\partial z} \\
-\kappa_{B} T_{i} \frac{\partial}{\partial z}\left[\sum_{j, q} n_{j, q}(z, t)\right]-\left(\sum_{j, q} m_{j} S_{j, q}^{0}(z, t)\right) u_{i}(z, t)
\end{array}
$$

can be solved to determine the plasma potential $\varphi(z, t) . T_{i}$ is the ion temperature, $m_{j}$ the ion mass and $S_{j, q}^{0}$ the ion source due to ionization/charge-exchange of neutral particles (not from other ion charge-states). This profile can then be used in the Fokker-Planck electron modeling. The procedure is iteratively performed until convergence.

The 1D ion modeling is further coupled to the 1D electron modeling by ambipolarity. The sheath potential at the device edge is adjusted to maintain equal ion and electron flows to the walls.

The neutral atoms in the plasma are treated using a simple particle balance scheme. The neutral density is determined by balancing the neutrals free flowing across the borders of each axial cell along with the number of neutrals lost to ionization or charge-exchange inside each cell. A more complex treatment, such as a Monte-Carlo 
3D neutral code isn't justified due to the uncertainties in the 3D plasma profiles. The neutral densities outside the plasma are estimated from the gas pressure measured as close as possible to the plasma chamber. When trying to match experimental data, this neutral density must be adjusted to account for the measurement location outside the plasma chamber and for the presence of many impurity partial pressures in a typical ECRIS.

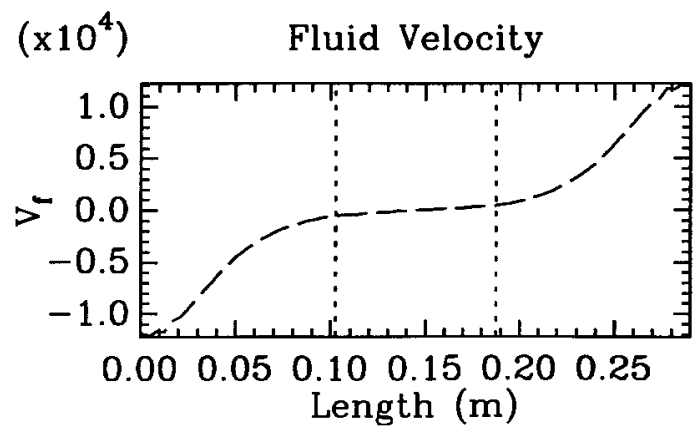

Figure 5: Ion fluid velocity profile (in $\mathrm{m} / \mathrm{s}$ ). Dotted lines show location of $\mathrm{rf}$ resonance.

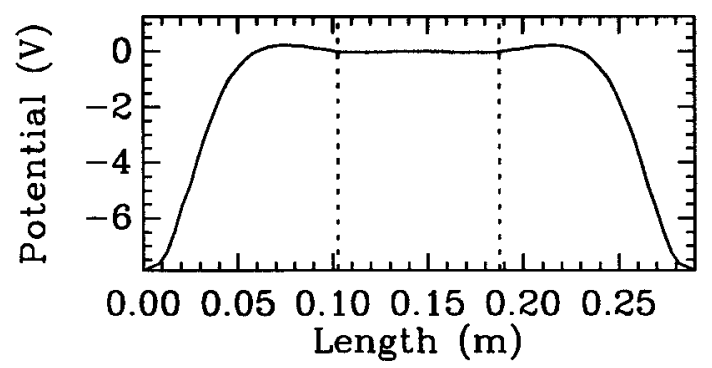

Figure 6: Plasma electrostatic potential profile. The model predicts a potential well inside the plasma core. Dotted lines show location of $\mathrm{rf}$ resonance.

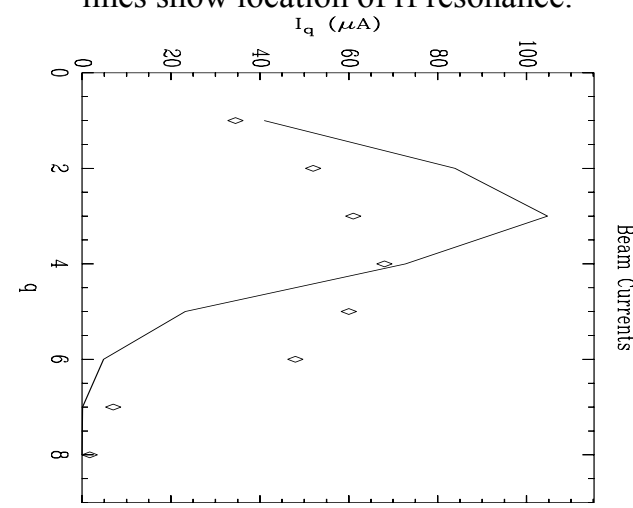

Figure 7: GEM predicted CSD (line) peaks at a lower charge than experimentally observed (diamond)

Results of the 1D fluid modeling are presented in Figures 5, 6 and 7. The modeling run is the same as shown for the electron modeling. Of particular note is the hollow potential well in the plasma core predicted by GEM. This potential well is believed to be a key feature of ECRIS ion confinement.

As discussed above, the 1D GEM predicts several typical ECRIS features that are impossible to determine from a $0 \mathrm{D}$ model. Unfortunately, the CSD predictions do not yet show good agreement with experimental data. Figure 7 shows model predictions along with experimental data from ECR-I [8] at ANL. Although, of the correct order of magnitude, the predicted CSD peaks at a lower charge state than experimentally observed. Several possible explanations for this discrepancy are being investigated:

- The assumption of a uniform fluid velocity may be inaccurate. Separate ion fluid velocities should result in higher charge states being more potentially confined allowing them more time to be ionized to higher charge states.

- The collision cross-sections used may need to be updated to include the most recent data.

- Uncertainties in the experimental data used, e.g. the ECRF power actually deposited in the plasma.

- The boundary conditions used in the 1D fluid model may not be appropriate.

To compare code predictions for the ion fluxes leaving the plasma with Faraday cup data, GEM numerical results can be used as initial conditions for an ion extraction code such as IGUN (2D) [9] or MICHELLE (3D) [10]. For ECRIS extraction studies, plasma sheath calculations have been implemented into the MICHELLE code.

Extraction modeling and experimental data indicates that the Faraday cup current CSD is subject to spacecharge effects which distorts the actual CSD of the ions flowing out of an ECRIS. To properly benchmark and validate GEM, data is needed on the actual parameters inside an ECRIS plasma. A measure of the core plasma CSD and EDF using, for example soft X-ray spectroscopy, would provide data that can be directly compared with modeling predictions.

The authors thank John Petillo for his help this work.

\section{REFERENCES}

[1] G. Shirkov and G. Zschornack, Nuc. Instrum. and Meth. in Phys. Res. B 95, 527 (1995).

[2] V. D. Dougar-Jabon, A. M. Umnov and V. B. Kutner, Rev. Sci. Instrum. 67, 1152 (1996).

[3] D. H. Edgell, J. S. Kim, S. K. Wong, R. C. Pardo and R. Vondrasek, Phys. Rev. Special Topics-Accelerators and Beams 2, 123502 (1999); D. H. Edgell, J. S. Kim, S. K. Wong, R. C. Pardo and R. Vondrasek, Rev. Sci. Instrum. 71, 666 (2000)

[4] M. Arnaud \& R. Rothenflug, Astron. Astrophys. Suppl. Ser. 60, 425 (1985)

[5] A. A. Mirin, M. G. McCoy, G. P. Tomaschke, and J. Killeen, Comp. Phys. Comm., 81, 403 (1994)

[6] T. A. Cutler, L. D. Pearlstein and M. E. Rensink, LLNL Technical Report \# UCRL-52233 (1977); T. D. Rognlien, LLNL Report \# UCID-20474 (1985)

[7] M. Schlapp, R. C. Pardo, R. C. Vondrasek, J. Szczech, P. J. Bilquist, J. Vieregg, Z. Q. Xie, C. M. Lyneis and R. Harkewicz, Rev. Sci. Instrum. 69, 631 (1998).

[8] D. P. Moehs, R. Vondrasek, R. C. Pardo, and D. Xie, Rev. Sci. Instrum. 71, 761 (2000).

[9] R. Becker and W. B. Herrmannsfeld, Rev. Sci. Instrum. 63, 2756 (1992).

[10] J. Petillo, RPAH090 in these proceedings. 